\title{
Deleting a marked state in quantum database in a duality computing mode
}

\author{
LIU Yang ${ }^{1,2}$ \\ ${ }^{1}$ School of Nuclear Science and Engineering, North China Electric Power University, Beijing 102206, China; \\ ${ }^{2}$ Department of Physics, Tsinghua University, Beijing 100084, China
}

Received February 23, 2013; accepted April 17, 2013; published online June 4, 2013

\begin{abstract}
In this article, we present a deletion algorithm in the duality computer that deletes a marked state from an even superposition of all basis-states with certainty. This duality computer deletion algorithm requires a single query, and this achieves exponential speedup over classical algorithm. Using a duality mode and recycling quantum computing, we provide a realization of this duality computer deletion algorithm in quantum computer.
\end{abstract}

duality computer, deletion algorithm, search algorithm, database, recycling quantum computing

Citation: Liu Y. Deleting a marked state in quantum database in a duality computing mode. Chin Sci Bull, 2013, 58: 2927-2931, doi: 10.1007/s11434-013-5925-9

Deleting items from a database has been a widely met scientific problem and it has important applications in computing process. In classical computing, deleting is an essential method to preserve certain data structure for convenient searching and visiting, for example, a well-known Google search engine PageRank algorithm [1]. Classical deleting is considered equivalent to classical searching. To delete $M$ marked items from a $N$-item database usually requires $O(M N \log N)$ steps. Fibonacci heaps algorithm [2] which has been shown optimal [3] is the optimal classical deletion algorithm. For arbitrary database, Fibonacci heaps algorithm deletes $M$ marked items from a $N$-item heap with $O(N \log N+M)$ steps. Recently, the deletion algorithm has been extended to quantum computation [4]. A quantum deletion algorithm is proposed that deletes a marked basis-state from an evenly distributed state with only a single query. Compared to its classical counterpart, quantum deletion algorithm can achieve an exponential speedup.

Duality computer (DC) is a recently proposed computing model [5]. It exploits particle wave duality property, and it may offer additional computing capability. Two proofof-the-principle designs of duality computer have been presented. Later on, many efforts have been made to duality

email: yliu@ncepu.edu.cn computer research. A duality computer can solve an unsorted database search problem [5] and factorization for large integers [6]. Mathematical theory of duality computer has also been worked out [7-15]. A quantum computer realization of the duality computer is given in [16], and complex duality quantum computer is proposed in [17]. This realization uses the duality mode and recycling quantum computing. These modes provide new ways and flexibility in quantum algorithm designs, for example, a fixed-point quantum search algorithm based on the duality mode is proposed [18], which can achieve speedup compared to previous fixed-point searching [19]. Later it is experimentally demonstrated [20]. An improved fixed-point duality quantum algorithm is given in [21]. Duality quantum computing has been generalized into more broad range, duality quantum information processing $[22,23]$. In this article, we explore the problem of deleting marked item using the duality computer, and the duality mode in quantum computer.

This article is organized as follows. In Section 1, we briefly review the duality computer. In Section 2, we briefly review the duality mode and the recycling quantum computing. In Section 3, we present a algorithm using the duality computer to delete a marked basis-state from an even superposition of all basis-states. In Section 4 , we give a quantum simulation of the deletion algorithm in Section 3 using the 
duality mode and recycling quantum computing. In Section 5 , a concluding remark is given.

\section{The duality computer}

Simply stated, a duality computer [5] is a moving quantum computer passing through different slits. A duality computer possesses duality parallelism which is absent in a quantum computer, and it offers the capability to perform separate operations on the sub-waves coming out of the different slits. In a duality computer, the basic information carrier is called duality bit for dubit for short, two new computing gates are quantum wave divider (QWD) and quantum wave combiner (QWC). Take a $m$-slit system in complex Hilbert space $H$ for example, copies of the wave function is produced by a QWD with coefficients $\sqrt{p_{i}}$ in $m$ direct summed Hilbert spaces $H^{\oplus m}=\sum_{i=1}^{m} \oplus H_{i}$, namely

$$
D_{p}(|\psi\rangle)=\sum_{i=1}^{m} \oplus \sqrt{p_{i}}|\psi\rangle_{i},
$$

where $p_{i}$ is called divider function which represents the strength of the sub-wave in the $i$-th slit and satisfies $\sum_{i} p_{i}=1$.

The quantum wave combiner (QWC) combines the subwaves in the $H^{\oplus m}$ into the Hilbert space $H$,

$$
C\left(\sqrt{p_{1}}\left|\psi_{1}\right\rangle \oplus \cdots \oplus \sqrt{p_{m}}\left|\psi_{m}\right\rangle\right)=\sum_{i=1}^{m} \sqrt{q_{i}} \sqrt{p_{i}}\left|\psi_{i}\right\rangle,
$$

where $\sqrt{q_{i}}$ is called combiner function. The following operations can describe a duality computing process:

$$
\begin{aligned}
|\psi\rangle & \rightarrow \sum_{i=1}^{m} \oplus \sqrt{p_{i}}|\psi\rangle_{i} \rightarrow \sum_{i=1}^{m} \oplus \sqrt{p_{i}} U_{i}|\psi\rangle_{i} \\
& \rightarrow \sum_{i=1}^{m} \sqrt{q_{i}} \sqrt{p_{i}} U_{i}|\psi\rangle
\end{aligned}
$$

Here is a summary of a symmetric 2-route duality computer, i.e. $q_{1}=q_{2}=1 / 2, p_{1}=p_{2}=1 / 2$. A quantum system with $n$ dubits is moving, its wave function is $|\psi\rangle=|\varphi\rangle|\kappa\rangle$, where $|\varphi\rangle$ is the internal state, $|\kappa\rangle$ is the center of mass translational motion function. Then a QWD operation is performed, the wave function becomes

$$
|\psi\rangle^{\prime}=\sum_{i=1}^{2} \oplus \sqrt{\frac{1}{2}}|\varphi\rangle\left|\kappa_{i}\right\rangle .
$$

Then different gate operations are performed on different subwaves,

$$
|\psi\rangle^{\prime \prime}=\sqrt{\frac{1}{2}} \sum_{i=1}^{2} \oplus U_{i}|\varphi\rangle .
$$

Then a QWC operation is performed and the wave function becomes

$$
|\psi\rangle_{f}=\sum_{i=1}^{2}\left(\frac{1}{2} U_{1}+\frac{1}{2} U_{2}\right)|\varphi\rangle .
$$

To read-out the information, perform a measurement on $|\psi\rangle_{f}$ state. The duality gate $\sum_{i=1}^{2} \frac{1}{2} U_{i}$ is no longer unitary. Quantum computer cannot perform $U_{1}+U_{2}$, it can only perform $U_{1} U_{2}$, so this is fundamental difference between duality computer and quantum computer.

\section{Duality mode and recycling quantum computing}

Duality mode and recycling quantum computing provide a quantum computer simulation of the duality computer [16]. An $n$-dubit duality computer can be simulated by an $(n+1)$ qubit quantum computer where one qubit is auxiliary qubit. There is following correspondence:

$$
\begin{aligned}
&|\varphi\rangle\left|\kappa_{u}\right\rangle \leftrightarrow|\varphi\rangle|0\rangle, \\
&|\varphi\rangle\left|\kappa_{d}\right\rangle \leftrightarrow|\varphi\rangle|1\rangle .
\end{aligned}
$$

If the auxiliary qubit is in $|0\rangle$ or $|1\rangle$ state, it simulates that a duality computer passes through the upper or lower slit respectively. Initial and final wave functions of the duality computer are ascribed to the auxiliary qubit that is in state $|0\rangle$. Thus the initial state of the duality computer is $|\varphi\rangle|0\rangle$. Perform the QWD to switch on the duality mode, and the state becomes

$$
|\varphi\rangle \frac{|0\rangle+|1\rangle}{\sqrt{2}}
$$

namely, the QWD operation is equivalent to a WalshHadamard operation on the auxiliary qubit. As shown in Figure 1 [16], conditional gates can simulate gate operations on different slits.

Then the wave function becomes

$$
\frac{U_{0}|\varphi\rangle|0\rangle+U_{1}|\varphi\rangle|1\rangle}{\sqrt{2}} .
$$

The QWC operation can be simulated by a Walsh-Hadamard operation on auxiliary qubit to switch off the duality mode. After QWC, the wave function becomes

$$
\frac{U_{0}+U_{1}}{2}|\varphi\rangle|0\rangle+\frac{U_{0}-U_{1}}{2}|\varphi\rangle|1\rangle .
$$

A measurement is performed on the $n$ qubits in the condition that the auxiliary qubit is in $|0\rangle$ state. Then the wave function is collapsed and $\left(U_{0}+U_{1}\right)|\varphi\rangle$ result is read out. The probability of obtaining a result is $P_{0}=\left\langle\varphi\left|\left(U_{0}+U_{1}\right)^{\dagger}\left(U_{0}+U_{1}\right)\right| \varphi\right\rangle / 4$.

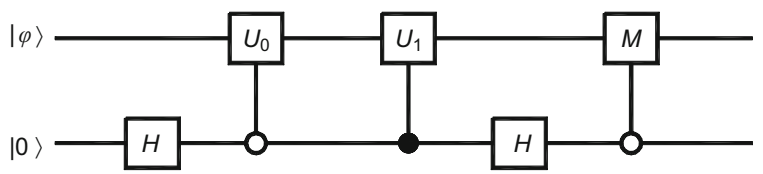

Figure 1 Schematic illustration of a duality mode. An $n$-dubit duality computer can be simulated by an $(n+1)$-qubit quantum computer in the duality mode. 
The probability of not obtaining a result is $1-P_{0}$ and if this occurs, the state in $|1\rangle$ collapses out, and the wave function becomes

$$
\left|\psi^{\prime}\right\rangle=N^{\prime} \frac{U_{0}-U_{1}}{2}|\varphi\rangle|1\rangle,
$$

where $N^{\prime}$ is renormalization constant.

As shown in Figure 2 [16], then a unitary recovering operation $V$ is performed on the $n$ qubits to restore the initial input state. Flip the auxiliary qubit state $|1\rangle$ to $|0\rangle$. The $(n+1)$ qubits are recovered to be the beginning of the circuit. The calculating process will recycle again and again until the conditional measurement is performed to obtain a result. This is the recycling quantum computing mode.

\section{Deletion algorithm using duality computer}

In quantum computing, a quantum deletion algorithm can delete a marked basis-state from an even superposition of $N$ basis-states with only a single query [4]. The deletion problem is:

Given: a function $f:[N] \rightarrow\{0,1\}$ as a black box.

Promise: there exists a unique $\tau$ such that $f(\tau)=1$.

Goal: map the state $\sum_{i=1}^{N}|i\rangle$ to $\sum_{i \neq \tau}|i\rangle$.

We can view an even superposition of all basis-states as an unsorted database like in the Grover algorithm [24], so we can view above problem as deleting a marked state from an unsorted database. For example, if we search for multiple marked states in an unsorted database and if one marked state has already been found, we can delete one marked state in subsequent search for other marked states. This problem can also be solved in duality computer, the duality computer algorithm is given as follows.

Step 1: Prepare duality computer state in the evenly distributed state,

$$
\left|\psi_{0}\right\rangle=\frac{1}{\sqrt{N}}(|0\rangle+|1\rangle+\cdots+|\tau\rangle+\cdots+|N-1\rangle),
$$

where $\tau$ is the marked state we want to delete.

Step 2: The duality computer go through QWD, so the wave is divided into two sub-waves:

$$
\begin{aligned}
& \left|\psi_{u}\right\rangle=\frac{1}{\sqrt{2 N}}(|0\rangle+|1\rangle+\cdots+|\tau\rangle+\cdots+|N-1\rangle), \\
& \left|\psi_{d}\right\rangle=\frac{1}{\sqrt{2 N}}(|0\rangle+|1\rangle+\cdots+|\tau\rangle+\cdots+|N-1\rangle) .
\end{aligned}
$$

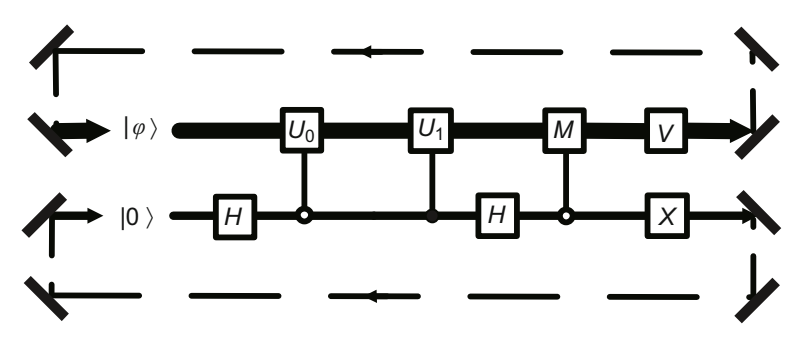

Figure 2 Schematic illustration of a recycling quantum computation.
Step 3: The query is applied to the lower-path sub-wave, reversing the coefficient of the marked state $|\tau\rangle$ and leaving all other basis-states untouched, and then the lower sub-wave becomes

$$
\left|\psi_{d}^{\prime}\right\rangle=\frac{1}{\sqrt{2 N}}(|0\rangle+|1\rangle+\cdots-|\tau\rangle+\cdots+|N-1\rangle) .
$$

No operation is applied to the upper-path sub-wave, and it remains in state eq. (13).

Step 4: The sub-waves are combined at QWC, and the wave becomes

$$
\left|\psi_{f}\right\rangle=\frac{1}{\sqrt{N}} \sum_{i \neq \tau}|i\rangle .
$$

Step 5: Make a read-out measurement, and the marked item $\tau$ can be deleted.

Compared to classical deletion, the duality version only requires a single query and achieves an exponential speedup. The query can be simulated using $O(\ln N)$ dubits. Namely, the difficulty in constructing the query increases only logarithmally as the size of the database $N$ increases.

\section{Deletion algorithm using duality mode in quantum computer}

The duality mode has provided a new avenue for algorithm design. Here we give an algorithm solving the problem in Section 1 using the duality mode in quantum computer. One can start from evenly distributed state,

$$
\frac{1}{\sqrt{N}} \sum_{i=0}^{N-1}|i\rangle|0\rangle,
$$

and perform a Walsh-Hadamard transform on the auxiliary qubit to switch on the duality mode; then the state becomes

$$
\frac{1}{\sqrt{2 N}} \sum_{i=0}^{N-1}|i\rangle(|0\rangle+|1\rangle) .
$$

In duality mode of quantum computer, let unitary $U_{0}$ and $U_{1}$ in Figure 2 be $U_{0}=I$ and $U_{1}=I-2|\tau\rangle\langle\tau|$. After two controlled gates, the wave function becomes

$$
\frac{1}{\sqrt{2 N}} \sum_{i=0}^{N-1}|i\rangle|0\rangle+\frac{1}{\sqrt{2 N}}\left(-|\tau\rangle+\sum_{i \neq \tau}|i\rangle\right)|1\rangle .
$$

Then recombine the two sub-waves through a WalshHadamard gate on the auxiliary qubit, we obtain

$$
\frac{1}{\sqrt{N}} \sum_{i \neq \tau}|i\rangle|0\rangle+\frac{1}{\sqrt{N}}|\tau\rangle|1\rangle .
$$

Then conditionally measuring on the auxiliary state being in $|0\rangle$, one obtains an even superposition of all basis-states except $\tau$ with probability $(N-1) / N$. This is simply achieved by 
measuring the auxiliary qubit. If it is 0 , then measure to delete $\tau$; this happens with probability $\frac{N-1}{N}$. If it is 1 , the state collapses to $\tau$; this happens with probability $1 / N$. This state can be reused again after a flipping on the auxiliary qubit and an unitary recovering operation to the original input state. The unitary recovering operation consists of successive recovering subroutines. Indicating the recovering subroutine as $S$, it contains four steps:

Step 1: Perform a $n$-qubit Walsh-Hadamard transform $W$, namely $W=H^{\otimes n}$.

Step 2: Perform a conditional phase shift $-e^{-i \phi}$ to $|0\rangle$ state and $e^{-i \pi}=-1$ to all other basis-states. Denoting this action as $-I_{0}$, and it is

$$
-I_{0}=-I-\left(e^{-i \phi}-1\right)|0\rangle\langle 0|,
$$

where $\phi$ will be given later in eq. (22).

Step 3: Perform a $n$-qubit Walsh-Hadamard transform $W$ again.

Step 4: Perform a conditional phase shift $e^{-i \phi}$ to the $|\tau\rangle$ state and leave all other basis-states untouched. The action is denoted as $I_{\tau}$,

$$
I_{\tau}=I+\left(e^{-i \phi}-1\right)|\tau\rangle\langle\tau| .
$$

In above steps, the two phases are equal, and this is required by the phase matching condition in quantum search algorithm [25-28]. Suppose the state of the $n$ working qubits is recovered to an even superposition of $N$ basis-states in $J$ times of subroutine, we have worked out explicitly the phase $\phi$ using the $S O(3)$ picture of quantum algorithm [29,30],

$$
\phi=2 \arcsin \left(\frac{\sin \frac{\pi}{4 J+2}}{\sin \beta}\right),
$$

where $\sin \beta=\sqrt{1 / N}$. Eq. (22) has real solutions for

$$
J \geqslant \frac{\pi}{4 \beta}-\frac{1}{2}
$$

Then the optimal iteration number is

$$
j_{\text {op }}= \begin{cases}j_{m} & \text { if } j_{m} \text { is an integer; } \\ \operatorname{INT}\left[j_{m}\right]+1 & \text { if } j_{m} \text { is not an integer }\end{cases}
$$

where

$$
j_{m}=\frac{\pi}{4 \beta}-\frac{1}{2},
$$

and INT[] means taking the integer part. In our quantum deletion algorithm using duality mode, the undesirable results is restored to the input state by $J_{\text {op }}$ times of $S$ iteration and guided to the input end. The calculation process is repeated again and again until we obtain the final result. The conditional phase rotation $\phi$ and optimal iteration number $j_{\mathrm{op}}$ versus the database size $N$ are given in Figures 3 and 4.

Hence we can see that, in order to restart the deletion, it will be easier to prepare the evenly superposed state than to

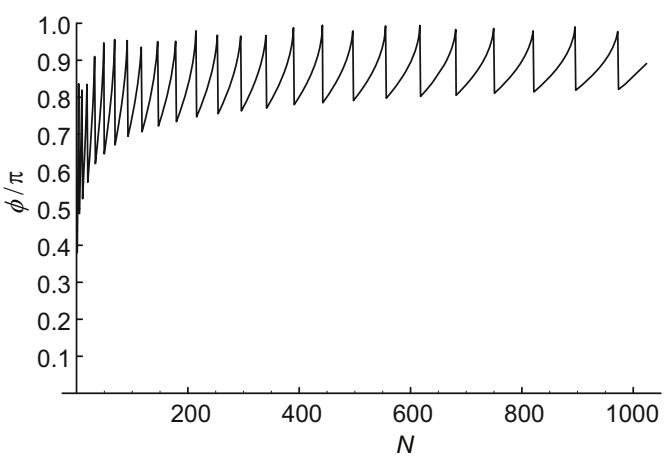

Figure 3 The conditional phase rotation $\phi$ versus $N=2^{n}$ in deletion algorithm.

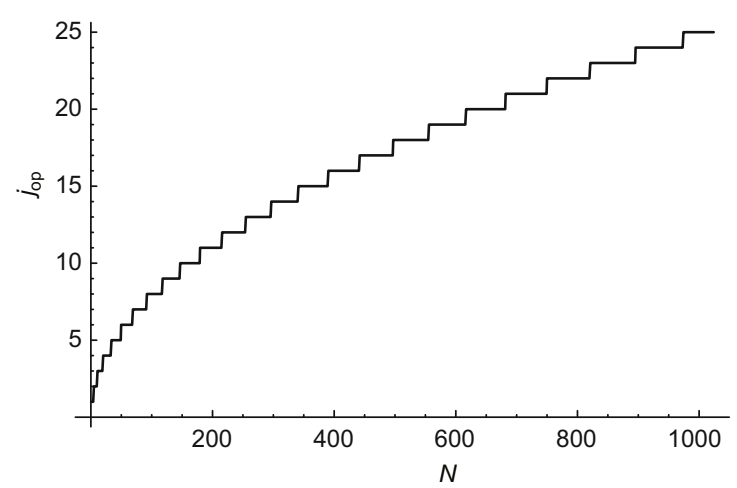

Figure 4 The optimal iteration number $j_{o p}$ versus $N=2^{n}$ in deletion algorithm.

recover it from the state $|\tau\rangle$. The recovery process is actually the reverse of the improved Grover algorithm with certainty [29]. The recovery process is beautiful mathematically; however it is not required in practice.

\section{Conclusions}

In summary, a duality computer deletion algorithm with high probability is presented. The duality computer deletion algorithm only requires a single query which achieves an exponential speedup over classical computation. Moreover, we show the quantum computer realization of this duality computer deletion algorithm using the duality mode. This realization is a fix-point deleting process, and itself serves as a new avenue in constructing duality computing algorithm.

This work was supported by the Fundamental Research Funds for the Central Universities.

1 Page L, Brin S, Motwani R, et al. The pagerank citation ranking: Bringing order to the web. Technical Report, Stanford Digital Libraries, 1998

2 Freadman M L, Tarjan R E. Fibonacci heaps and their uses in improved network optimization algorithms. J Assoc Comput Mach, 1987, 34: $596-615$ 
3 Abuaiadh D, Kingston J H. Are Fibonacci heaps optimal? In: Proceedings of Algorithms and Computation 5th International Symposium, 1994. 442-450

4 Liu Y, Long G L. Deleting a marked basis-state from an even superposition of all basis-states with a single query. Int J Quant Inform, 2009, 7: 567-572

5 Long G L. The general quantum interference principle and the duality computer. Commun Theor Phys, 2006, 34: 825-844

6 Wang W Y, Shang B, Wang C, et al. Prime factorization in the duality computer. Commun Theor Phys, 2007, 47: 471-473

7 Gudder S. Mathematical theory of duality quantum computers. Quant Inf Proc, 2007, 6: 37-48

8 Long G L. Mathematical theory of the duality computer in the density matrix formalism. Quant Inf Proc, 2007, 6: 49-54

9 Du H K, Wang Y Q, Xu J L. Applications of the generalized Kuders theorem. J Math Phys, 2008, 49: 013507

10 Guo Z H, Cao H X. Existence and construction of a quantum channel with given inputs and outputs. Chin Sci Bull, 2012, 57: 4346-4350

11 Guo Z H, Cao H X, Chen Z L, et al. Operational properties and matirx representations of quantum measures. Chin Sci Bull, 2011, 56: 16711678

12 Cao H X, Li L, Chen Z L, et al. Restricted allowable generalized quantum gates. Chin Sci Bull, 2010, 55: 2122-2125

13 Cui J X, Zhou T, Long G L. An optimal expression of a Krauss operator as a linear combination of unitary matrices. J Phys A: Math Theor, 2012, 45: 444011

14 Cao H X, Chen Z L, Guo Z H, et al. Complex duality quantum computers acting on pure and mixed states. Sci China Phys Mech Astron, 2012, 55: 2452-2462

15 Zhang Y, Cao H X, Li L. Realization of allowable generalized quantum gates. Sci China Phys Mech Astron, 2010, 53: 1878-1883

16 Long G L, Liu Y. Duality computing in quantum computers. Commun
Theor Phys, 2008, 50: 1303-1306

17 Long G L, Liu Y, Wang C. Allowable generalized quantum gates. Commun Theor Phys, 2009, 51: 65-67

18 Long G L, Liu Y. Duality quantum computing. Front Comput Sci China, 2008, 2: 167-178

19 Grover L K. Fixed-point quantum search. Phys Rev Lett, 2005, 95: 150501

20 Hao L, Long G L. Experimental implementation of a fixed-point quantum search algorithm in the nuclear magnetic resonance quantum system. Sci China Phys Mech Astron, 2011, 54: 936-941

21 Hao L, Liu D, Long G L. An N/4 fixed-point duality quantum search algorithm. Sci China Phys Mech Astron, 2010, 53: 1765-1768

22 Long G L. Duality quantum computing and duality quantum information processing. Int J Theor Phys, 2011, 50: 1305-1318

23 Li C Y, Wang W Y, Wang C, et al. Duality quantum information and duality quantum communication. AIP Conf Proc, 2011, 1327: 158-165

24 Grover L K. A fast quantum mechanical algorithm for database search. In: Proceedings of 28th Annual ACM Symposium on Theory of Computing, 1996. 212-219

25 Long G L, Zhang W L, Li Y S, et al. Arbitrary phase rotation of the marked state can not be used for Grover's quantum search algorithm. Commun Theor Phys, 1999, 32: 335-338

26 Long G L, Li Y S, Zhang W L, et al. Phase matching in quantum searching. Phys Lett A, 1999, 262: 27-34

27 Long G L, Xiao L, Sun Y. Phase matching condition for quantum search with a generalized quantum database. Phys Lett A, 2002, 294: 143-152

28 Hoyer P. The phase matrix. In: Proceedings of 16th International Symposium on Algorithms and Computation, 2005. 308-317

29 Long G L. Grover algirhtm with zero theoretical failure rate. Phys Rev A, 2001, 64: 022307

30 Long G L, Tu C C, Li Y S, et al. An $S O(3)$ picture for quantum searching. J Phys A, 2001, 34: 861-866

Open Access This article is distributed under the terms of the Creative Commons Attribution License which permits any use, distribution, and reproduction in any medium, provided the original author(s) and source are credited. 